\title{
Analysis on the use of Latent Semantic Indexing (LSI) for document classification and retrieval system of PNP files
}

\author{
Angelica M. Aquino*, and Enrico P. Chavez \\ Technological Institute of the Philippines-Manila, Philippines
}

\begin{abstract}
Document classification is the process of categorizing documents from many mixed files automatically [1]. In this paper, an approach to classification of documents for admin-case files of Philippine National Police (PNP) using Latent Semantic Indexing (LSI) method is proposed. The model for this that represents term-to-term, document-todocument and term-to-document relationships has been applied. Regular Expression is implemented also to define a search pattern based on character strings which the LSI used to establish the semantic relevance of the character strings to the search term or keyword. The aim of the study is to evaluate the performance of LSI in classifying PNP documents; experimentation was done using software to test the capability of LSI towards text retrieval. Indexing is according to the pattern matched in the collection of text that uses model of SVD. Based on tests, documents were indexed based on file relationships and was able to return a search result as the retrieved information from PNP files. Weights are used to check the accuracy of the method; the positive values identified in query similarity are regarded as the most relevant among the related searches, meaning, the query word matches words in a text file and it returns a query result.
\end{abstract}

\section{Introduction}

It is necessary for software to have the ability to classify file before it will be stored. Document classification is a scheme of identifying the category of business activities and the records they generate as files used to facilitate retrieval mechanism [2]. Moreover, Delgado [2] stated that records are group together for the following reasons: (1) to keep documents of a similar theme together; (2) to make it easier to find the information we are looking for; and (3) to provide a context for individual documents and records. By applying a technique in classifying documents for retrieval purposes, documents can be searched systematically and LSI is the suggested method to perform document classification and retrieval system. In relation to this, it is important to consider a technique for classifying and storing files, because having an intelligent system of a file storing would definitely help an organization to handle documents without suffering the confidentiality of the information. With the need of PNP to handle efficiently the documents for admin-case information, a classification technique for documents shall be implemented. This solves the

* Corresponding author: aaquino@letran-calamba.edu.ph 
dilemma of PNP in categorizing and searching admin-case files. To resolve the dilemma of an incorrect result of searching, a technique for document retrieval is proposed.

According to Barto [3], a tool for retrieving information is the use of techniques that search documents which are stored from a computer and it searches a document that exists in a collection of documents from a group of full-text database. As mentioned by Barto [3], a full-text search usually performs an examination of all the words that exist in the documents stored in the attempt of matching criteria of searching. In this paper, the admincase files of PNP which are stored in a.pdf file format is converted to a txt file for Information Retrieval (IR) purposes. Rasjid et al.[4], said that, by using IR, it is possible to obtain information and automatic classification. These text files are used for document categorization which is based on the keyword used in pattern searching. The performance of a full-text search is applied here and the process of retrieving information usually begins with a query from a user into the system. Query is a formal statement indicating the need for particular information [5]. The relevance of available information is determined depending on the technique used with respect to the entered queries and the results given are based on their relevance to the queries.

\section{Review of related literatures}

Cavanagh [6] defined document classification as content-based assignment of one or more predefined categories to documents and it usually applies machine learning to build classifiers automatically. The application of machine learning in document classification can optimize document search activities. In fact, machine learning can perform key phrase extraction, language and topic detection which provides capability of a system to let users search files. This idea has been adopted in this experimental study to prove the capability of LSI.

According to Ponweiser [7], Latent Dirichlet Allocation (LDA) is an information retrieval model solvable by machine learning techniques. This model is seen as classical text mining or natural language processing tools that support information retrieval. LSI is used here to optimize the retrieval technique that indexes and uses SVD that identifies the pattern in an unstructured collection of text and find relationship between patterns [8]. Regex is used to define a search pattern based on the character strings. This pattern is then used by LSI to establish the semantic relevance of the character strings to the search term (keyword). In this study, this concept is used to show how document-to-document, term-toterm, and term-to-document relationships performs to show the semantic relevance of the searched strings. This has been used in testing the SVD in querying.

In addition, LSI can be used on query documents [9] and it has emerged as a competitive text retrieval technique [10]. Thus, this research explores the use of Regex to define a search pattern based on character strings. According to Bui et al. [11], a regular expression is a sequence of characters that describes a pattern of text. Also, regular expression-based classifier can be combined with other classifiers like SVD to improve classification performance as it provides a term-document matrix. LSI performs the conversion of document in index into a vector of word occurrences. The number of dimensions of a vector is equal to the number of unique words in the entire document set where this vector is scaled so that every term reflects the frequency of its occurrence to form a term-document matrix [1]. Rows represent terms, columns represent documents and it performs SVD on the term-document matrix [10]. Furthermore, according to one study, LSI has been used in web technology as the search engines are developed to retrieve accurate information [12]. Also, LSI has been used as clustering algorithm in medical documents as it includes acronyms of clinical data [11]. In view of this, LSI can be applied to cluster admin-case documents of PNP as it contains case-related acronyms. 
To improve the retrieval process of LSI, stop words are implemented to identify commonly used words which will be ignored out before or after processing of a natural language (text files). Examples are articles (e.g., $a$, an, the), prepositions (e.g., at, by, in, to, from, with) and conjunctions (e.g., and, but, as, because) [13]. As mentioned by Zaman et al. [14], removal of stop words plays an important role in different text processing domains and it can be used to get language relations [15]. This was attested by Shin et al. [16], as they mentioned that stop word removal improves the quality of the indexed terms.

\section{Methodologies}

The study used experimental approach of research to test the theories mentioned regarding document classification using LSI through the attainment of valid conclusions about the analysis set in the study. To perform the test and to analyse the capability of LSI, data for this experiment was obtained from PNP and these are admin-case files. These files are kept in a . $p d f$ file format and it contains different but related documents about a case. In applying the method of LSI, case files in .pdf format is converted first into a text file (test data) before performing the test. A program is created to test LSI and it was executed in Python 2.7 with its plug-ins such as Numpy 1.11 for array manipulation and SciPy 0.18.1 for data models [17]. The program which performs document classification and information retrieval, LSI implements LSA which uses SVD and returns the result for document similarity, term similarity, and query similarity. To show the performance of LSI, see figure 1 for the process of testing.

Figure 1 show the command prompt (cmd) that uses a sample code in running LSI executed in Python 2.7.

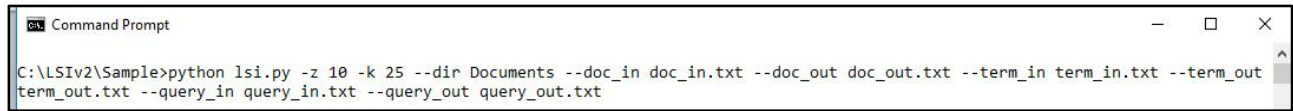

Fig. 1. Running Latent Semantic Indexing (LSI) Program in Python 2.7

In running the Latent Semantic search engine, LSI program uses this code (syntax): $-z$ 10 - $k 25$--dir Directory --doc_in $<$ name of input document file $>--$ doc_out $<$ name of output document file to be generated by code $>--$ term_in<name of input term file $>-$-term_out $<$ name of output term file to be generated by code $>$--query_in $<$ name of input query file $>$-- query_out $<$ name of output query file to be generated by code $>$ In actual (written in Figure 1):

-z 10 -k 25 --dir Documents --doc_in doc_in.txt -- doc_out doc_out.txt -- term_in term_in.txt --term_out term_out.txt --query_in query_in.txt -- query_out query_out.txt where:

-z: Dimensionality of lower dimensional space

-k: \# of similar terms/documents to be returned --dir: Directory containing input documents --doc_in: Input file containing list of document titles (one per line) corresponding to whom $k$ similar documents are to be returned.

--doc_out: Each line of this file will have titles of $k$ documents (separated by '; <tab $>$ ' i.e semicolon followed by tab) that are similar to the document in the corresponding line of doc_in --term_in: Input file containing list of words (one per line) corresponding to whom $k$ similar words/terms are to be returned.

--term_out: Each line of this output file will have $k$ words (separated by '; $<$ tab $>$ ' i.e semicolon followed by tab) that are similar to the word in the corresponding line of term in

--query_in: Input file containing list of queries (one per line) corresponding to whom $k$ relevant documents are to be returned.

--query_out: Each line of this output file will have titles of $k$ documents (separated by '; $<$ tab $>$ 'i.e semicolon followed by tab) that are relevant to the query in the corresponding line of query_in

\section{Results and discussion}


The performance of the program which is executed in Python 2.7 implements the following: (1) given a document title, it outputs $k$ similar documents (document-to-document); (2) given any word, it outputs $k$ related words from all the documents and if this word occurs in none of the documents it outputs $k$ random words (term-to-term); and (3) given a query, it outputs $k$ relevant documents for the query (term-to-document). The performance has been optimized using SVD and stop words to work well in large cases. Moreover, it was demonstrated that LSI performs key phrase extraction, language and topic detection which provides capability of a system to let users search files.

To evaluate the performance of LSI, fifty (50) text files (which contain information about the case) were used as test data in this experiment. It contains name of case, case number, and the person/s involved in the case. Given a sample query (query_in.txt), name of case was used as keyword/s and the result of search is written in the query result (query_out.txt).For instance, the query_in.txt file contains $A W O L$ as the searched keyword, and then the quey_out.txt will have the listing of PNP files as the search results that matches the keyword AWOL. From this context, LSI is capable of classifying documents towards information retrieval.

To demonstrate the retrieving process, see table 1 for the sample keywords used in searching a file. The test data used returns a query results for term-to-document relationships. query_in is used as input (search keyword) and query_out as output (search result).

Table 1 show example keyword/s used in the experiment and its Search Result Output.

Table 1. Keywords Used in Searching and Search Result.

\begin{tabular}{|c|l|l|}
\hline $\begin{array}{c}\text { Search } \\
\text { Keyword }\end{array}$ & \multicolumn{1}{|c|}{ Admin Case Name } & \multicolumn{1}{|c|}{ Search Result } \\
\hline AWOL & $\begin{array}{l}\text { Serious Neglect of Duty } \\
\text { (Absence Without Official Leave) }\end{array}$ & $\begin{array}{l}\text { Returns admin case files with } \\
\text { AWOL case (listed in query_out.txt); } \\
\text { or read the text files which } \\
\text { contain the word } A W O L \text { and } \\
\text { return the searched file/s. }\end{array}$ \\
\hline $\begin{array}{c}\text { Physical } \\
\text { Injuries }\end{array}$ & $\begin{array}{l}\text { Grave Misconduct (Serious } \\
\text { Physical Injuries, Threat and Gun } \\
\text { Toting) }\end{array}$ & $\begin{array}{l}\text { Returns admin case files with } \\
\text { Physical Injuries case (listed in } \\
\text { query_out.txt); or the text file } \\
\text { contain the two-term Physical } \\
\text { Injuries and return searched } \\
\text { file/s. }\end{array}$ \\
\hline $\begin{array}{c}\text { Rude } \\
\text { Insulting }\end{array}$ & $\begin{array}{l}\text { Simpuage } \\
\text { or insulting language or exhibit } \\
\text { similar rudeness to the public) }\end{array}$ & $\begin{array}{l}\text { Returns admin case files with } \\
\text { Rude Insulting Language case } \\
\text { (listed in query_out.txt); or the text } \\
\text { file contain the three-term } \text { Rude } \\
\text { Insulting Language and return } \\
\text { the searched file/s. }\end{array}$ \\
\hline
\end{tabular}

The query_in text file contains the keyword for searching and the query_out text file contains the output for the retrieved PNP files (file names of case files in pdf format) after running the program of LSI (lsi.py). Furthermore, the model for representing the documentto-document and term-to-term relationships was also recognized and LSI was able to perform its process for information retrieval. The result of using doc_in and doc_out, term_in and term_out was implemented in the performance of LSI to determine the relationships of different test data. For document-to-document relationship, it was tested that if the input given is the name of case file ( $d o c_{-}$in) it shows an output of different files with its case title of $k$ documents which are similar to the documents that correspond to the 
line of doc in. For term-to-term relationship, it was tested that if the input given is a word (term_in) it shows an output of $k$ words similar to the word that correspond to the line of term_in given in the input file.

To check the accuracy of the proposed method, the weights are used to identify the most relevant in related searches. Meaning, all positive weights are the searched item/s where keyword used was matched in the searching. For example in query similarity output of LSI, there are positive and negative weights (i.e $0.27145569795,-0.0034859544576$ ) generated by the program for the search results. The searched output is sorted by related searches where positive weights are the most relevant. For instance, the input query $A W O L$ returns related documents as the most relevant searches based on positive weights. A negative weight shows non-related documents to the query word $A W O L$, meaning it does not match the word $A W O L$ from the group of text files in PNP documents.

\section{Conclusion}

Based on the experiment done, LSI with SVD is capable of performing document indexing and retrieval for an information given. The relationships of test data (PNP case files) have been proved to show the capability of LSI through document-to-document, term-to-term, and term-to-documents operations. Moreover, LSI performs with the consideration of a high value of $k$. The search result as retrieved information relies on the number of $k$ set in document searching. A very low value of $k$ gives poor results since it only returns few files as retrieved information, meaning $k$ value should be higher. Therefore, the low and high percentage for determining the number of retrieved information depends on $k$ values. The highest value set for $k$ affects the high percentage of retrieved information. In addition, LSI performs the SVDs calculation and returns an output for document similarity, term similarity, and query similarity. More importantly, as the result of test, the performance of LSI is accurate based on the generated weights of query similarity where a positive value signifies the most relevant searches. Meaning, relevant searches are documents related to the searched keyword. The capability of LSI executed in Python thus proved to be beneficial in document indexing and retrieval of information which was applied in admincase files of PNP. To increase the precision of the document classification process, other research in this matter is suggested: (1) Advance research on comparison of web information or other information to be classified; and (2) Further research on the use of other indexing algorithms and other metrics used for accuracy of the indexing method.

\section{References}

1. Ronghui Ju, Pan Zhou, Cheng Hua Li, and Lijun Liu. (2015). An Efficient Method for Document Categorization Based on Word2vec and Latent Semantic Analysis.Computer and Information Technology; Dependable, Autonomic and Secure Computing; Pervasive Intelligence and Computing (CIT/IUCC/DASC/PICOM), 2015 IEEE International Conference on. 10.1109/CIT/IUCC/DASC/PICOM.2015.336

2. Carmen Delgado. (2012). File Classification Scheme for Administrative Functions Common to all UN Offices. United Nations.

3. A. Barto, et al. (2015). Learning to Act Using Real-Time Dynamic Programming.Hoboken: Rutledge Press pp.112-123.

4. Z. Rasjid, and R. Setiawan. (2017). Performance Comparison and Optimization of Text Document Classification using k-NN and Naïve Bayes Classification Techniques. 2nd International Conference on Computer Science and Computational Intelligence 2017, ICCSCI 2017, 13- 14 October 2017, Bali, Indonesia 
5. M. Malki. (2016). "Comprehensive Study and Comparison of Information Retrieval Indexing Techniques" International Journal of Advanced Computer Science and Applications (IJACSA), 7(1), 2016. http://dx.doi.org/10.14569/IJACSA.2016.070120

6. L. Cavanagh. (2016). Optimizing document search using Machine Learning and Text Analytics [BlogPost]. Microsoft Azure. Retrieve January 5, 2018 from https://azure.microsoft.com/en-us/blog/optimizing-document-search-using-machinelearning-text-analytics

7. Martin Ponweiser. (2012). Latent Dirichlet Allocation in R. Institute for Statistics and Mathematics.Diploma Thesis. Retrieved December 10, 2017 from http://epub.wu.ac.at/3558/1/main.pdf

8. H. Chen., B. Martin, C. Daimon, and Maudsley, S. (2013).Effective use of latent semantic indexing and computational linguistics in biological and biomedical applications. $\quad$ Front. $\quad$ Physiol., 30 January 2013 https://doi.org/10.3389/fphys.2013.00008

9. Kumar B Shravan, and V. Ravi. (2017). One-Class Text Document Classification with OCSVM and LSI. In: Dash S., Vijayakumar K., Panigrahi B., Das S. (eds) Artificial Intelligence and Evolutionary

10. Ashwini Deshmukh, and Gayatri Hegde. (2012). A Literature Survey on Latent Semantic Indexing. International Journal of Engineering Inventions ISSN: 2278-7461, www.ijeijournal.com Volume 1, Issue 4 (September 2012) PP: 01-05

11. Duy Duc An Bui, and Zeng-Treitler. (2014). Learning Regular Expressions for Clinical Text Classification. J Am Med Inform Assoc. 2014 Sep; 21(5): 850-857. Published online 2014 Feb 27. doi: 10.1136/amiajnl-2013-002411

12. S. Hingmire, et.al. (2013). Document Classification by Topic Labeling. Conference: International Conference on Information Retrieval (SIGIR 2013), At Dublin, Ireland, Volume: pp. 877 - 880, ACM Press

13. A. Janusz, W. Świeboda, A. Krasuski, and H. Nguyen, (2012). Interactive Document Indexing Method Based on Explicit Semantic Analysis. J.T. Yao et al. (Eds.): RSCTC 2012, LNAI 7413, pp. 156-165, 2012. c Springer-Verlag Berlin Heidelberg

14. A N K Zaman, Pascal Matsakis, and Charles. Brown. (2011).Evaluation of Stop Word Lists in Text Retrieval Using Latent Semantic Indexing. 978-1-4577-1539-6/ IEEE

15. A.P. SivaKumar, P. Premchand, and A. Govardhan. (2011). Application of Latent Semantic Indexing for Hindi-English CLIR Irrespective of Context Similarity. In: Wyld D.C., Wozniak M., Chaki N., Meghanathan N., Nagamalai D. (eds) Trends in Network and Communications. Communications in Computer and Information Science, vol 197. Springer, Berlin, Heidelberg

16. JH Shin, M. Abebe, C.J Yoo, S. Kim, J.H Lee, and HK Yoo. (2017). Evaluating the Effectiveness of the Vector Space Retrieval Model Indexing. In: Park J., Pan Y., Yi G., Loia V. (eds) Advances in Computer Science and Ubiquitous Computing. CSA 2016, CUTE 2016, UCAWSN 2016. Lecture Notes in Electrical Engineering, vol 421. Springer, Singapore

17. Scott Shell. (2014). An Introduction to Numpy and Scipy. Retrieved January 5, 2018 from https://engineering.ucsb.edu/ shell/che210d/numpy.pdf 